\title{
Sensors Synergistic With Nature For In-pile Nuclear Reactor Measurements
}

FIIW 2012

James A. Smith

Dale Kotter

Steven L. Garrett

Randall A. Ali

U.S. Department of Energy

National Laboratory

operated by

Battelle Energy Alliance

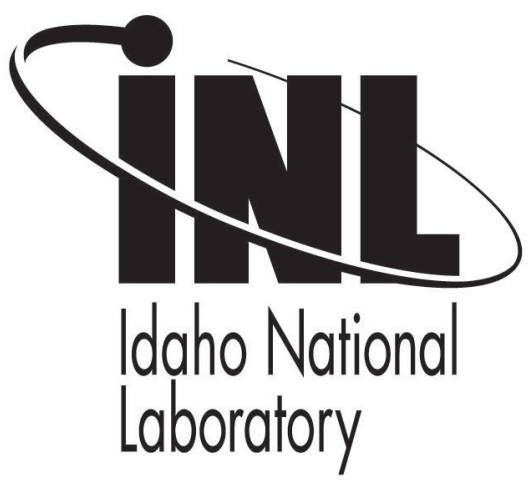

This is a preprint of a paper intended for publication in a journal or proceedings. Since changes may be made before publication, this preprint should not be cited or reproduced without permission of the author. This document was prepared as an account of work sponsored by an agency of the United States Government. Neither the United States Government nor any agency thereof, or any of their employees, makes any warranty, expressed or implied, or assumes any legal liability or responsibility for any third party's use, or the results of such use, of any information, apparatus, product or process disclosed in this report, or represents that its use by such third party would not infringe privately owned rights. The views expressed in this paper are not necessarily those of the United States Government or the sponsoring agency. 


\section{Sensors Synergistic With Nature For In-pile Nuclear Reactor Measurements}

\author{
James A. Smith, Dale Kotter \\ Fundamental Fuel Properties \\ Idaho National Lab \\ Idaho Falls, ID USA \\ James.Smith@INL.gov
}

\author{
Steven L. Garrett, Randall A. Ali \\ Graduate Program in Acoustics \\ Penn State University \\ State College, PA USA
}

\begin{abstract}
To be able to evolve fuel and structural microstructure within a nuclear power reactor in an engineered manner, an effective extreme environment sensor must exist. The development of sensor technology for nondestructive and nonintrusive measurements in harsh environments is a very active field. However most of the effort has been in adapting existing sensing technology to meet the harsh environmental requirements. A different approach is being presented. The fundamental question that we are trying to answer is how do we take advantage of the harsh environment and maintain synergy between the sensor and the environment. This paper will discuss the synergistic sensor being developed that takes advantage of harsh environments.
\end{abstract}

Index Terms-Sensor, acoustics, thermoacoustics, synergistic, microstructure, fission gas, nuclear, nondestructive, wireless, thermal, self powered.

\section{INTRODUCTION}

Advancing the Nuclear Fuel Cycle and Next Generation Nuclear Power Plant requires enhancing our fundamental understanding of fuel and materials behavior under irradiation. The two most critical issues limiting the performance and lifetime of the fuel are the degradation of the thermal conductivity of the fuel and the mechanical integrity of both the fuel and cladding.

To better understand thermal conductivity we must have the means to perform high fidelity in-core fuel temperature measurements. Several technology barriers exist for implementation of sensors into reactor vessels, especially if near core in high neutron flux regions. To overcome these barriers the devices must include: 1) high temperature durable materials and sensors that can operate up to $1100{ }^{\circ} \mathrm{C} ; 2$ ) Radiation hardened materials and electronics; and 3) Wireless communication methods compatible with harsh environments. Wireless RF signals encounter large attenuation through the coolant medium and much backscatter/noise from the reactor vessel housing. Thus Novel data transmission methods will be required to expand the transmission range and data rate of communication with in-situ sensors.

To better understand mechanical integrity of the fuel we must have the means to perform nondestructive analysis of the material. An efficient fuel must have the right microstructure within the reactor to perform properly. It is proposed that the evolution of defects in microstructures can change the overall material porosity. This research demonstrates that changes in porosity can be detected with the thermoacoustic effect and remotely monitored as a change in acoustic resonance frequency. Microstructure changes in fuel can be monitored directly by sensing changes in porosity or indirectly by monitoring fission gas release. Initial experimentation has been performed using surrogate materials, representative of fuels/cladding, with precisely controlled porosity. Preliminary experiments have also been performed using different gasses and pressures within the sensor. The effects of porosity on heat transfer and mechanical integrity can then be further studied and used as the basis for better understanding the thermo mechanical performance of the fuel.

Instead of pursuing the adaption of existing technology, it was decided that a paradigm shift was necessary to develop effective sensors for the harsh nuclear environment. The fundamental question that should drive sensor design is how the harsh environment can be used to the sensors advantage. Instead of working against the environmental conditions, how can the sensor be synergistic and take advantage of its environment as well as Mother Nature.

This research introduces a thermoacoustic (TAC) $[1,2]$ sensor that enables both high temperature and porosity sensing capability within a nuclear environment. The TAC requires no mechanical pump, moving parts, or external power source to provide a wireless indication of temperature. TAC sensors work synergistically with harsh in-pile environments and can potentially be incorporated within current cladding and capsule designs for in-pile use. The sensor is self-powered by the temperature differential between the fuel and the coolant.

Since the thermoacoustic device can be constructed from robust materials and has no moving parts, thermoacoustic devices are ideally suited for the hostile conditions within a reactor.

\section{GENERAL THEORY OF OPERATION}

Thermoacoustics can be defined as the generation of acoustic waves from heat transfer. A TAC engine, which produces acoustic energy at a specific pitch, consists of heat 
sources and sinks, resonator, and "stack" (i.e., a porous medium that mediates the conversion of heat flow to sound) as shown in Figure 1. The heat sources and sinks provide the thermal gradient necessary to generate the acoustic wave and pumps a jet due to acoustic streaming [2] within the working fluid. The resonator provides the confinement and feedback to generate pressure oscillations. This effect is analogous to the optical laser [1].

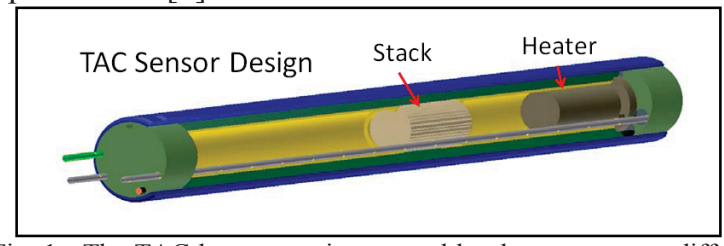

Fig. 1. The TAC heat pump is powered by the temperature differential across the stack. In this embodiment, the stack is Celcor ${ }^{\circledR}$, a ceramic material, usually used as a substrate for automotive catalytic converters, with 1,100 parallel square pores per square inch.

The thermoacoustic engine converts heat from a hightemperature source into acoustic power, while rejecting waste heat to a low-temperature sink (i.e., coolant). When the acoustically-driven gas moves through the stack, the pressure, temperature, and position of each gas parcel oscillates with time at the resonance frequency. When the gas is enclosed within a resonator tube, sound bounces back and forth that creates an acoustic standing wave. The temperature variation imposed on the stack drives heat into the gas forcing it to do work on its surroundings and amplifying the acoustic oscillations. Each parcel of oscillating gas takes heat from the one behind and transfers it to adjacent gas molecules; effectively acting as a heat exchanger. Maintenance of the steep thermal gradient required to induce thermoacoustic operation requires an external source of power. For laboratory testing, the TAC device is powered by an electrically-resistive $\mathrm{NiCr}$ heater element, but in a reactor environment fuel will be used as the power source.

\section{EXPERIMENTAL PLAN}

Testing for direct and indirect measure of microstructure will be performed. The direct measure of microstructure will be performed by inserting porosity specimens into the TAC's resonance cavity within the hot section. The porosity specimens consist of a honey combed steel puck that has been immersed in wax. The wax blocks the pores making a nonporous specimen. When the specimen is placed in the TAC sensor, the wax melts and runs out of the porosity specimen. Resulting modulation to the acoustic signal will be evaluated.

The indirect measurement of microstructure will be simulated by filling the resonant cavity with different gasses. Thermal acoustics can be used to measure gas composition [3-5]. In fact, the most precise value for the Universal Gas Constant was determined from the measurement of acoustical resonances at the National Institute of Standards and Technology [6]. It is proposed that future advanced TAC designs will be optimized to measure the rate of production of fissile gases krypton and xenon which are influenced by microstructural changes [7].
For different gases, the speed of sound is inversely dependent on square root of the mean molecular weight of the gas, and affected to a lesser extent by the number of ways in which the molecules of the gas can store heat from compression, since sound in gases is a type of compression. In low molecular weight gases, such as helium, sound propagates faster compared to heavier gases, such as xenon. For a given ideal gas, the sound speed depends only on its absolute (Kelvin) temperature. At a constant temperature, the ideal gas pressure has no effect on the speed of sound, because pressure and density (also proportional to pressure) have equal but opposite effects on the speed of sound and the two contributions cancel out exactly. Experiments were performed with nitrogen, helium, xenon, and atmospheric air.

\section{RESULTS AND DISCUSSION}

The initial results from the wax porosity measurements are looking promising. The direct porosity results are shown in Fig. 2. The salient phenomenon demonstrated in this graph is that the movement of the melting wax perturbed the cavity such that the sonic resonance was extinguished. Once the melted wax stopped flowing the sonic resonance started back up but at a slightly higher frequency. The theory to describe the changes in the cavity is under development.

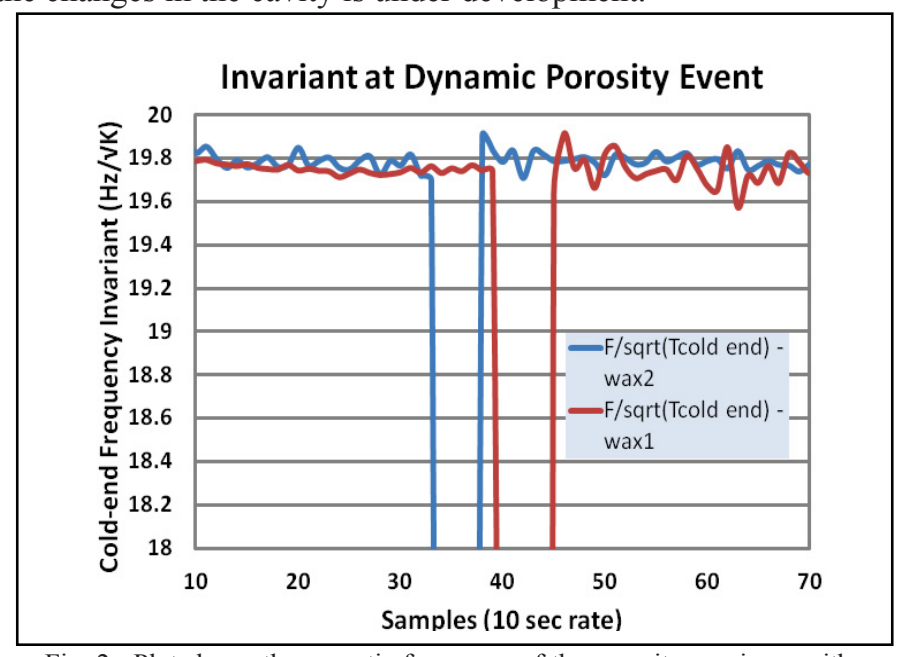

Fig. 2. Plot shows the acoustic frequency of the porosity specimen with time. As the porosity specimen heats up, the wax melts. The flowing wax keeps the TAC sensor from resonating and the signal drops out near $15 \mathrm{Sa}$. Once the liquid wax has finished flowing, the cavity is stabilized and the sound resonates again at a slightly higher frequency.

The simulated fission gas experiments are also promising as shown in Fig.3. Experimental results validated that resonance frequency is directly correlated to the mean molecular mass of the gas. Varying the gas density of the TAC resulted in changes in the pitch and the amplitude of the sound radiated based on the molecular weight of the gas. Although "dry" air has a molecular weight larger than nitrogen, the presence of humidity in the air dropped the average molecular weight below nitrogen. Thus the TAC sensor can also be a humidity sensor if properly configured. 


\section{CONCLUSIONS}

A novel sensor based on the Thermal Acoustic phenomena has been presented. The TAC sensor is shown to be synergistic with Mother Nature within a nuclear reactor. TAC technology takes advantage of the large thermal gradients caused by the nuclear fuel and surrounding cooling water. Because the phenomenon is simplistic and has no moving parts, the sensor can be constructed from inexpensive yet robust materials. The signal generated is acoustic and thus is transmitted easily through the cooling water or metal pipes/structures within the reactor.

The experiments performed were designed to demonstrate the microstructure testing potential of the TAC sensor. Two types of microstructure monitoring have been tested. Direct monitoring of pores opening and indirect measure of porosity from fission gas release have been demonstrated.

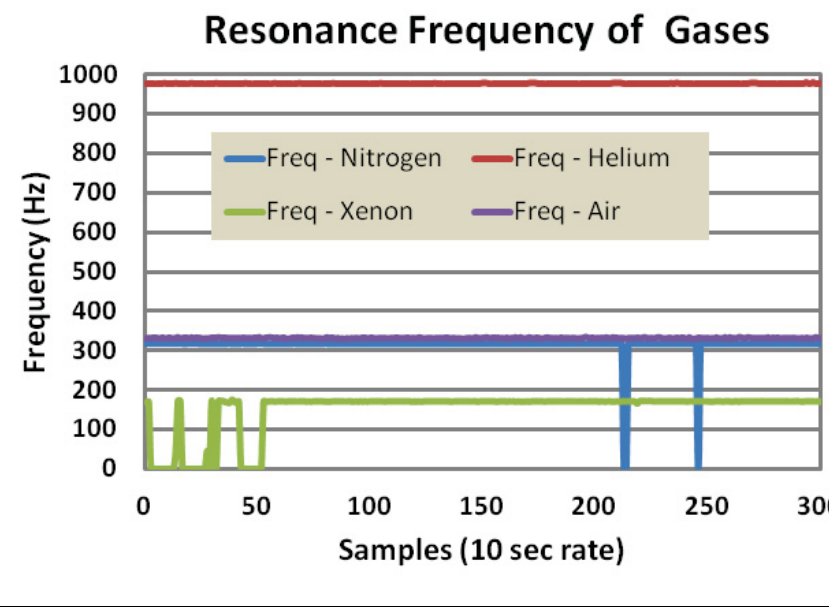

Fig. 3. Plot showing the experimental results which validate that resonance frequency is directly correlated to the mean molecular mass of the gas. The resulting frequencies are properly ordered based on molecular weight.

The TAC sensor has been demonstrated to monitor the opening of pores as the filler material melted and drained.
The TAC sensor also detected a change in the final state of the resonant cavity. The TAC sensor properly ordered based on molecular weight and differentiated these gasses: nitrogen, helium, xenon, and atmospheric air. The TAC sensor has sufficient fidelity to clearly differentiate between nitrogen and atmospheric air. Since the TAC sensor is robust, wireless, self-powered and economical, the TAC sensor is an excellent choice for in-pile monitoring within a nuclear reactor. The TAC sensor has high potential because it is in harmony with nature.

\section{ACKNOWLEDGMENT}

The authors would like to acknowledge their project sponsors: DOE NE, DOE ID, INL and Pennsylvania State University. The authors would also like to acknowledge the wonderful design support from Rick Parry.

\section{REFERENCES}

[1] S. L. Garrett and S. Backhaus, "The power of sound," American Scientist 88(6), 516-525 (2000); "Le son transformé en froid," Pour la Science (French-language version of Scientific American), Dossier N 32, 112-117 (Juil/Oct 2001).

[2] G. W. Swift, Thermoacoustics: A unifying perspective for some engines and refrigerators (Acoust. Soc. Am., 2002); ISBN 0-7354-0065-2.

[3] M. V. Golden, R. M. Keolian and S. L. Garrett, "Sonic gas analyzers," Proc. 16th Int. Congress on Acoustics and 135th Meeting Acoust. Soc. Am., Vol. III, pp. 1705-1706. Seattle, WA, 20-26 June 1998.

[4] E. Polturak, S. L. Garrett, and S. G. Lipson, "Precision acoustic gas analyzer for binary mixtures," Rev. Sci. Inst. 57, 2837-2841 (1986).

[5] S. Garrett, G. W. Swift, and R. E. Packard, "Helium gas purity monitor for recovery systems," Physica 107B, 601-602 (1981).

[6] M. R. Moldover, et al., "Measurement of the universal gas constant $\mathfrak{R}$ using a spherical acoustic resonator," J. Research NBS 93, 85-144 (1988).

[7] W. M. Stacey, Nuclear Reactor Physics, 2nd ed., (Wienheim: Wiley \& Sons Inc., 2007). 